\title{
Historia
}

\section{La incidencia del Consejo Internacional de Enferme- ras en la historia y socialización de la Enfermería}

\section{The International Council of Nurses incidence for the history and socialization of Nursing}

O impacto do Conselho Internacional de enfermeiros na história e socialização dos enfermeiros

\author{
Taka Oguisso
}

Licenciada en Enfermería y abogada. Doctora en Salud Pública y Profesora Titular (catedrática) de la Escuela de Enfermería de la Universidad de São Paulo, Brasil. Presidenta de la Academia Brasileña de Historia de la Enfermería. Perteneció al staff del CIE, como directora ejecutiva adjunta, de 1987-1998.

Cómo citar este artículo en edición digital: Oguisso, T. (2012) La incidencia del Consejo Internacional de Enfermeras en la historia y socialización de la Enfermería. Cultura de los Cuidados. (Edición digital) 16, 32. Disponible en: http://dx.doi. org/10.7184/cuid.2012.32.04

Correspondencia: Taka Oguisso. Departamento de Orientação Profissional, da Escola de Enfermagem da Universidade de São Paulo. Avda. Dr. Eneas de Carvalho Aguiar, 419. Cerqueira Cesar. Sao Paulo SP. Brazil. CEP: 05403-000. Email: takaoguisso@vol.com.br Recibido: 20/11/2011 - Aceptado: 12/2/2012

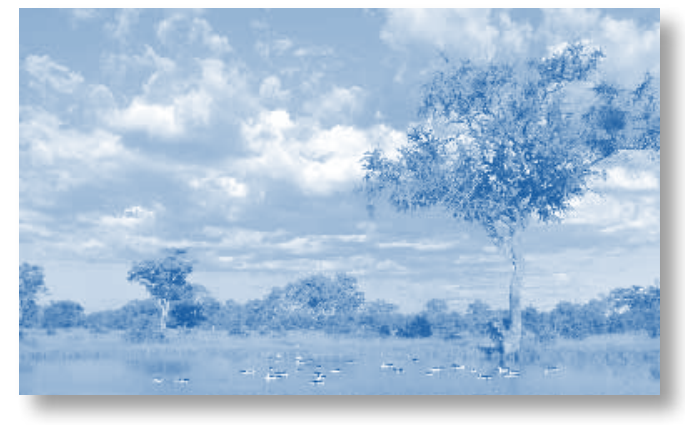

\section{ABSTRACT}

The International Council of Nurses (ICN) is a federation of national nurses associations (NNA), created in 1899 , by a small group of visionary women, in a milieu of enormous social changes such as industrial revolution and the slavery abolition and with woman's social rights, including the right for voting, social progressivism and healthcare reform. Against all odds, ICN has survived for over 100 years, due to dedication of three women: Ethel Bedford Fenwick, Lavinia Dock y Agnes Karll who created the very first international health professionals organization. The ICN incidence was very instrumental as nursing was just giving its first steps after being institutionalized as a profession by Florence Nightingale. The ICN diffusion and socialization will also benefit the History of Nursing as a field of study. Being the nurses' voice ICN can make their profession to develop and reach out wider geographical boundaries and to learn better their own profession and the nursing professional identity.

Key words: nursing history, history of the International Council of Nurses.

\section{RESUMO}

O Conselho Internacional de Enfermeiros (ICN) é uma federação de organizações nacionais de enfermagem, criada em 1899 por um pequeno grupo de mulheres visionário em 
meio a enormes mudanças sociais, como a revolução industrial e da abolição da escravatura, bem como a luta pela direitos das mulheres, social progressismo e reforma da saúde. Apesar de todas as crenças contrário, ICN sobrevive por mais de 100 anos desde que foi fundada, principalmente por três mulheres que se dedicam: Ethel Bedford Fenwick, Lavinia Dock e Agnes karll que criou a primeira organização internacional de profissionais de saúde. A incidência de ICD foi fundamental como a enfermeira estava dando seus primeiros passos após a institucionalização da profissão feita por Florence Nightingale. A divulgação e socialização da CIE será benéfico também para a História da Enfermagem, como campo de estudo. Como titular da voz enfermeiras podem crescer e chegar a um muito mais amplo limites geográficos para aumentar o conhecimento sobre sua própria profissão e da identidade profissional da enfermagem.

Palavras-chave: história da história da enfermagem, do Conselho Internacional de Enfermeiros.

\section{RESUMEN}

El Consejo Internacional de Enfermeras (CIE) es una federación de organizaciones nacionales de enfermería, creada en 1899, por un pequeño grupo de mujeres visionarias en medio a enormes cambios sociales tales como la revolución industrial y la abolición de esclavitud además de la lucha por los derechos de la mujer, el progresivismo social y la reforma de la salud. A pesar de todas las creencias al contrario, el CIE sobrevive por más de 100 años desde que fue fundado básicamente por tres dedicadas mujeres: Ethel Bedford Fenwick, Lavinia Dock y Agnes Karll que crearon la primera organización internacional de pro- fesionales de salud. La incidencia del CIE fue instrumental pues la enfermería estaba dando sus primeros pasos después de la institucionalización de la profesión hecha por Florencia Nightingale. La difusión y socialización del CIE será beneficiosa también para la Historia de la Enfermería, como un campo de estudios. Como porta-voz de los enfermeros podrá hacer crecer y alcanzar límites geográficos mucho más amplios para que conozcan mejor su propia profesión y la identidad profesional de enfermería.

Palabras clave: historia de la enfermería, historia del Consejo Internacional de Enfermeras.

\section{INTRODUCCIÓN}

El Consejo Internacional de Enfermeras (CIE) es una federación de organizaciones nacionales de enfermería admitidas formalmente como miembros. Tiene como misión representar a los enfermeros de todo el mundo, hacer avanzar la profesión e influir en las políticas de salud. Son sus objetivos: influir en la política de enfermería, de salud, social, y en las normas profesionales y socioeconómicas en todo el mundo; ayudar a las asociaciones nacionales de enfermería (ANEs) y a la competencia de las enfermeras(os); promover el fortalecimiento de las ANEs; representar a las enfermeras y a la enfermería en el plano internacional; establecer, recibir y gestionar fondos y fideicomisos que contribuyan al progreso de la enfermería y del CIE.

Así se justifica conocer un poco más sobre esta organización, entender su política de acción y las motivaciones que la sustentan como institución representativa de los enfermeros de todo el mundo y la voz de la enfermería en el plano internacional. 
El CIE es económica y políticamente independiente, autónoma, no partidarista ni gubernamental, compuesta por más de 135 asociaciones nacionales de enfermería (ANEs) y representa a más de dos millones de enfermeros. Su sede propia se encuentra en Ginebra, Suiza.

La organización está dirigida por una Junta Directiva de 15 miembros, procedentes de todos los continentes que componen las siete regiones del CIE, elegidos para un mandato de cuatro años y se reúne una vez al año, por cuatro a cinco días en su sede, Ginebra. La preside un Director Ejecutivo y un staff de aproximadamente 14 a 15 funcionarios fijos (de ellos 4 a 5 son enfermeros y otras son secretarias y archivadoras); hay además algunos especialistas contratados por plazo determinado para desarrollar proyectos específicos.

El mayor órgano de deliberación del CIE es el Consejo de Representantes Nacionales (CRN) que se reúne una vez cada dos años para decidir sobre las políticas de la organización, aprobación de cuentas, establecimiento de cotizaciones, admisión de nuevos miembros y elección de los miembros de la Junta Directiva. Cada ANE elige un(a) representante, que puede ser el propio presidente(a), algún miembro de la Junta Directiva local o cualquier otra persona que sea enfermero(a), acreditado por la ANE. Cada organización nacional puede enviar un representante oficial y uno o dos suplentes para el CRN, sin embargo, cada ANE independientemente del tamaño, tiene derecho a un solo voto.

Además del CRN, cada cuatro años, se realiza un congreso cuadrienal, en el cual pueden participar todos los enfermeros que estén afiliados a la ANE que es miembro del CIE. La presidenta del CIE para el periodo 2009-2013 es la enfermera Rosemary Bryant, de Australia.

\section{Orígenes históricos}

La historia de los primeros 65 años del CIE, de 1899 a 1964, fue publicada en 1967, por Daisy Bridges, Directora Ejecutiva de la organización en el periodo de 1948 a 1961. En verdad, Ethel Bedford Fenwick y Margaret Breay escribieron esta historia por primera vez, cubriendo el periodo de 1899 a 1925, con recopilaciones de datos de documentos oficiales y fue publicado en 1931. En 1989, Sheila Quinn escribió otro libro intitulado "ICN- past and present" (El CIE - pasado y presente), dándose continuidad a aquella historia de Bridges y también porque quería dejar registrada las iniciativas y la visión de las enfermeras-líderes y pioneras, para que las nuevas generaciones puedan inspirarse y llevar adelante este ideal hacia el futuro. Finalmente, Barbara Brush y Joan E. Linaugh, con otras cuatro colaboradoras de Inglaterra, Holanda, Canadá y Estados Unidos publicaron un nuevo libro en 1999 Enfermeras de todas las naciones - sobre la historia del Consejo Internacional de Enfermeras, con motivo del primer centenario del CIE.

El objetivote este trabajo es describir sobre la importancia de tener una organización específica de enfermería a nivel mundial, que representa la profesión junto a organizaciones internacionales, tales como la Organización Mundial de la Salud (OMS), la Organización Internacional del Trabajo (OIT), agencias internacionales de la Organización de las Naciones Unidas (ONU), como UNICEF, UNESCO y otras como la Cruz Roja Internacional; que promueva eventos científico-culturales para el intercambio de enfermeros de todo el mundo; y actúe como su portavoz frente a grandes plenarios anuales. Esta difusión y socialización de la historia del CIE será también beneficiosa para la Historia de la Enfermería y para todos 


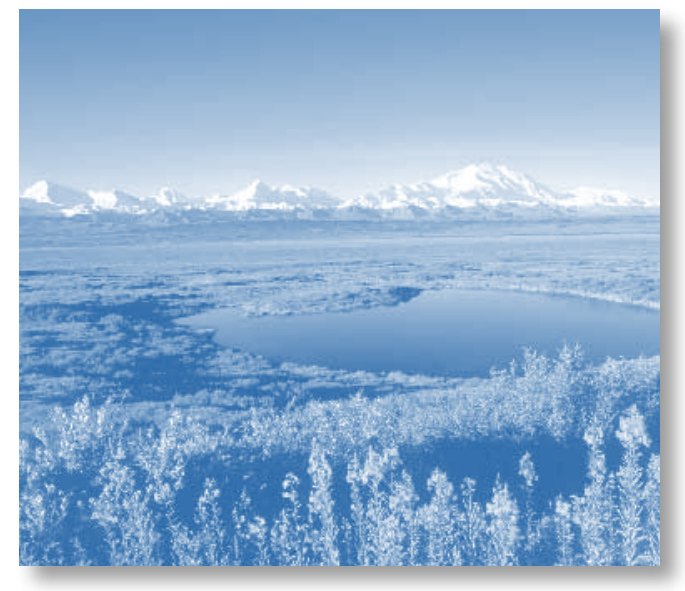

los que se dedican a estudiar este campo del saber, una vez que la incidencia del CIE ocurrió cuando la profesión daba sus primeros pasos después de la institucionalización hecha por Florencia Nightingale.

El Consejo Internacional de Enfermeras fue fundado el $1^{\circ}$ de julio de 1899 , en la ciudad de Londres. Parecería que todo empezó a partir de esa fecha, sin embargo no fue así. Es necesario retroceder un poco más para sentir el clima de la época y el contexto en el que fue creada esta organización.

En 1848, surgió en Estados Unidos de América (EUA) un clamor general de parte de las mujeres que querían igualdad de derechos educacionales, profesionales, políticos y de trabajo. Tal movimiento empezó primero en una convención realizada en Séneca Falls, una pequeña ciudad en el Estado de Nueva York. Como resultado de esta convención, aunque haya sido 40 años después, fue convocada una conferencia en el Teatro de Opera, en la ciudad de Washington, capital de los EUA, en marzo de 1888. Fue la primera conferencia del Consejo Internacional de Mujeres (CIM), asistida por 49 delegadas de once países y patrocinada por la Asociación Nacional del Sufragio Femenino que ya venía actuando extensamente a favor del derecho de voto de la mujer. El CIM convocaba a las mujeres de todo el mundo para que se unieran para intercambio de ideas y discutir nuevos y efectivos métodos para asegurar la igualdad y la justicia que las mujeres buscaban desde hace mucho tiempo.

La idea de fundar una organización internacional partió de una enfermera inglesa, Sra. Ethel Bedford Fenwick (1857-1947), que era muy activa en el Consejo Internacional de Mujeres y en enfermería, pues había fundado, en 1888, la Asociación Británica de Enfermería. No se puede olvidar que en esta época se vivía plenamente el periodo pos-Revolución Industrial y de la abolición de la esclavitud, y las mujeres ya sentían la injusticia social por no tener el derecho al voto ni cualquier poder político o social.

Después de la conferencia de 1888, la reunión siguiente se llevó a cabo en la ciudad de Londres, en 1899. Ethel Fenwick fue nombrada miembro de la comisión de finanzas de ese evento, además de responsable de la organización de una comisión profesional. En esta comisión creó la sección de enfermería con un día para discutir asuntos de la profesión. En la reunión participaron enfermeras famosas de la época, entre las cuales Lavinia Dock (18571956), de Estados Unidos, Isla Stewart de Gran Bretaña y Grace Neil ${ }^{1}$, de Nueva Zelanda. Esta Conferencia recibió una carta de Florencia Nightingale considerado como el último mensaje que dirigió personalmente a un grupo internacional de enfermeras.

Terminada la Conferencia del CIM, se realizó la Conferencia del Consejo de Matrons (denominación dada en Inglaterra a la directora de servicios de enfermería), presidida por Isla Stewart. Fue en este escenario, lleno de entusiasmo por la oportunidad de tener un encuentro para discutir cuestiones de enfermería, que surgió la idea de crear un órgano 
internacional. Había necesidad de que enfermería estuviera organizada y que las enfermeras pudiesen tener un sistema de educación y un método para el control de la profesión. Se pensaba que los principios deberían ser los mismos para todos los países, pues la necesidad de progreso era común a todos los pueblos. La propuesta de Ethel era la creación de un Consejo Internacional de Enfermeras en los mismos moldes del Consejo Internacional de Mujeres, con representantes de organizaciones de enfermería de cada país. Esa institución internacional debería auxiliar, en primer lugar, en la creación y organización de asociaciones o consejos de enfermería en cada país, donde no existiera un órgano propio de enfermería. La propuesta fue aceptada con entusiasmo por las participantes formándose una comisión en la cual Margaret Breay fue elegida como secretaria y tesorera. Las participantes británicas fueron solicitadas a redactar una constitución, que se hizo circular entre todas las participantes, para que en la reunión del año siguiente fuera aprobada. Es curioso destacar que en esa reunión la representante de Dinamarca, Sra. Gordon Norrie, propuso el inglés como idioma oficial de la nueva organización.

\section{LA CUESTIÓN DE LOS IDIOMAS}

Se abre un paréntesis para discutir la cuestión de los idiomas en el CIE, asunto siempre polémico y emocional, que acarreó muchas luchas y disputas. El inglés fue, de hecho, adoptado como idioma oficial y así permanece hasta hoy, y desde 1967 junto a otros dos idiomas de trabajo, el francés y el español, cuando se produjo la reforma de la Constitución del CIE que pasó a regir a partir del Congreso Cuadrienal, realizado en Montreal, en 1969. Enfermeros procedentes de países que no hablaban inglés expresaron sus dificultades para discutir en un forum internacional como el del CIE, en un idioma que no era el propio. Aún en la Constitución, enmendada en el 2009², el inglés continúa como idioma oficial, así entendido el idioma en que los documentos jurídicos del CIE y los archivos son mantenidos. En caso de alguna duda sobre el texto del documento traducido, prevalece el significado del texto original, en inglés. Los idiomas de trabajo, francés y español, son utilizados para traducir los documentos que se usan en reuniones del CRN y enviados a las asociaciones miembros de los países que hablen tales idiomas o cuando la organización miembro tenga optado por ese idioma.

El francés se incluyó como resultado de los reclamos de enfermeros franceses ${ }^{3}$ y canadienses pues en la primera mitad del siglo $\mathrm{XX}$ era considerado como idioma universal. Ya el español fue adoptado como tercer idioma al verificarse, en 1965, que la representación de enfermeros procedentes de países de habla española era mayor que el de francés. El CIE sólo tuvo una presidenta de idioma nativo español, la licenciada enfermera Nelly Garzon, de Colombia, en el período de 1985 a 1989. Para enfermeros oriundos de países que no hablan ninguno de estos tres idiomas del CIE (caso de Brasil, Japón, Italia, Tailandia, Egipto, etc) que son la absoluta mayoría entre sus asociaciones miembros, no hace ninguna diferencia, pues siempre estarán obligados a optar por uno de estos tres idiomas, teniendo de este modo que manejar y comunicarse con el CIE en un idioma que no es el propio. Sin embargo, asumen indirectamente el costo de la traducción e interpretación. Finalmente, en 1985, representantes de las asociaciones, francesa y canadiense, pidieron que la discusión sobre idiomas retornara y que se permitiera la inclusión del francés y del español como 
idiomas oficiales. Esta resolución fue aprobada en 1989, con validez a partir de las elecciones de 1993. Así, a partir de ese año, la Junta Directiva del CIE con quince personas, siempre cuenta con representantes de lengua española y/o francesa y las reuniones tienen traducción simultánea en los tres idiomas.

Cabe destacar que la Organización de las Naciones Unidas (ONU) considera, también, como oficiales esos tres idiomas, además del ruso, chino y árabe. El alto costo de la traducción e interpretación simultánea de esos idiomas en documentos, publicaciones y en las reuniones, imposibilita la mayor utilización de ese recurso, que sin duda, facilitaría una mejor comunicación entre los miembros.

\section{Funcionamiento inicial}

Cerrándose el paréntesis con relación al problema de los idiomas, retómese la historia del CIE nuevamente a partir de 1900, cuando la primera Constitución fue aprobada. Esta institución recién creada debería aceptar la afiliación como miembros a las organizaciones nacionales de enfermeros, sólo una por país siendo uno de los criterios de admisión que los enfermeros que componían la asociación fuesen formados en cursos de tres años de duración. Posteriormente este criterio tuvo que ser modificado, pues ni todos los países tenían cursos de enfermería con esta duración. El principio de aceptar solamente una organización representativa por país era justificado porque se consideraba que una institución poderosa, con la mayoría de los enfermeros unidos dentro de ella, era mejor que si existiesen varias organizaciones pequeñas, cada una representando parcelas de enfermeros. En verdad, este principio fue implementado a partir de 1904, pero sigue en pleno vigor hasta la Constitución actual del CIE. Por lo tanto, an- tes de 1904, la afiliación podía ser de enfermeros de forma individual o de organizaciones, si existiesen. Hoy, sólo las organizaciones de ámbito nacional pueden ser afiliadas y los enfermeros pertenecen indirectamente al CIE por medio de sus respectivas entidades nacionales.

Ethel Fenwick fue elegida como primera Presidenta de la nueva organización, Lavinia Dock como secretaria y la canadiense, Agnes Snively, como tesorera. Un espacio cedido por la Sociedad para el Registro Estatal de Enfermeras, con sede en Londres, fue la primera instalación del CIE. En la reunión de 1900, la Asociación de Enfermeras de Buffalo (EUA) invitó a la nueva organización a realizar un congreso de enfermería en esta ciudad americana. La invitación fue aceptada y el congreso se realizó en julio de 1901. Después de este congreso poco se pudo hacer teniendo al Océano Atlántico separando a la presidenta y a la secretaria y sin los medios de comunicación y transporte más ágiles, como los que existen hoy. En este primer momento sólo Estados Unidos, Gran Bretaña, Irlanda (del Norte) y Alemania habían oficializado su afiliación al CIE. Después, ingresaron Dinamarca, Canadá, Finlandia y Holanda, en 1904. En la década de 1910 fueron India y Nueva Zelanda, y en la década de 1920, Italia, Bélgica, China, Noruega, África del Sur, Bulgaria, Cuba, Francia, Irlanda, Polonia, Brasil, Grecia, Filipinas, Suecia y Yugoslavia. Varios de esos países se desligaron, sobre todo, debido a situaciones de guerra o revolución en sus países, pero después regresaron. Por ejemplo, Cuba ingresó antes que Brasil, en 1925, pero después de la revolución cubana en 1959, se retiró del escenario internacional, retornando solamente en 1981. Ya Brasil ingresó en 1929 y mantuvo ininterrumpida afiliación a través de la Asociación Brasileña de Enfermería (ABEn) hasta 1997 cuando 
pasó a ser representado por el Consejo Federal de Enfermería (COFEN). Otras ANEs de la Península Ibérica y América Latina fueron admitidas al CIE como puede verse a seguir:

1953 Chile

1957 Colombia, Panamá, Uruguay

1961 México, Venezuela

1965 España, Perú

1969 Portugal, Ecuador, Bolivia, Argentina, El Salvador, Costa Rica*

1973 Nicaragua

1977 Paraguay, Honduras, Puerto Rico ${ }^{\star *}$

1981 Cuba, Guatemala

1991 República Dominicana

${ }^{*}$ Costa Rica ingresó en 1969, después se retiró y fue admitida de nuevo en 1989.

${ }^{* *}$ Puerto Rico fue admitido por el CRN de 1975, pero sólo ingresó oficialmente durante el Congreso Cuadrienal de 1977, en Japón. Se retiró en la década de 1990 porque en un plebiscito el pueblo votó para que su país se quedara como Estado Asociado de los Estados Unidos. Como el CIE ya tenía una representación americana, con derecho al voto, no podía mantener la de Puerto Rico, de acuerdo con el principio constitucional.

Asociaciones de países involucrados en el conflicto de la 2a . Guerra Mundial (Japón, Alemania, Italia, Austria, Polonia, etc) también se retiraron del CIE y volvieron en 1949, al término de la guerra. Otros países como Checoslovaquia y Yugoslavia desaparecieron y con ellos desaparecieron las respectivas ANEs, surgiendo en su lugar las asociaciones de enfermería de la República Checa y la de Eslovaquia, resultante del desmembramiento de la antigua Checoslovaquia. Eslovenia, Croacia y Macedonia son de los países que emergieron de la antigua Yugoslavia, cuyas ANEs también se afiliaron al CIE. Se encuentran en proceso de filiación Moldavia, Senegal y Dominica. Otras entidades, como la de Irán, que se afiliara al

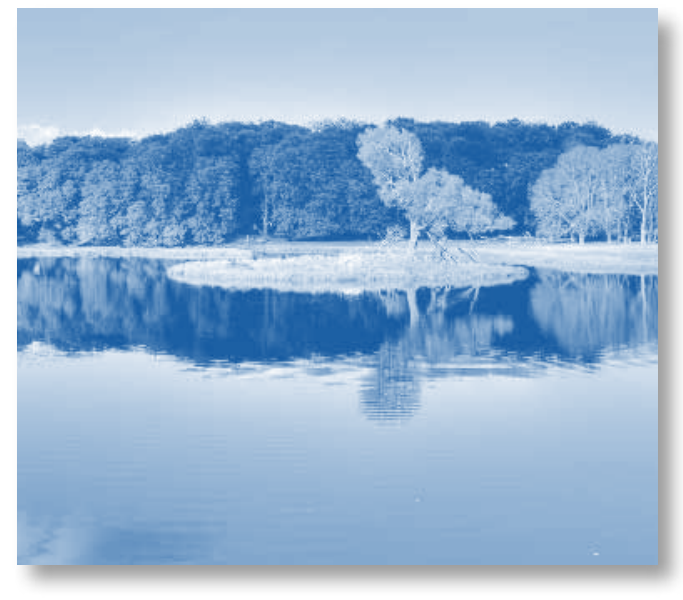

CIE en 1957, tuvieron que retirarse debido a problemas políticos en su país.

Todos estos aspectos son discutidos y votados en el CRN, cuando las líderes de cada país deciden los rumbos de la organización, la toma de decisión política por el CIE, así como la prestación de cuentas, elección de nuevos miembros de la Junta Directiva y otras cuestiones internacionales.

\section{Actividades internacionales}

Como se ha referido anteriormente, hasta 1904 la afiliación de los enfermeros al CIE podía hacerse individualmente y también de las ANEs, si estas ya existiesen. Desde el inicio de su fundación, el CIE dependió exclusivamente del trabajo voluntario de enfermeras dedicadas.

En 1909, el congreso ocurrió en la ciudad de Londres y fue elegida como presidenta del CIE una legendaria figura alemana de la época, Sra. Agnes Karll, que había fundado la Asociación Alemana de Enfermería, en 1903. Florencia Nightingale ya estaba muy enferma y debilitada y la presidenta pidió que el CRN concordara con el envío de una carta para ella, que fue respondida por una prima suya. En esa reunión de Londres, los participantes resolvieron presionar para que se crearan órganos 
de control y fiscalización en todos los países, contra el cual hubo una manifestación de médicos y autoridades de los hospitales. En esa reunión se incentivó, también, el trabajo de las enfermeras para la obtención del derecho de voto, como un derecho de ciudadanía, en los respectivos países, alabando a las que habían conquistado este derecho, como el caso de las finlandesas, en 1905.

El congreso siguiente fue en la ciudad de Colonia, Alemania, en 1912. En este Congreso, por primera vez los hombres enfermeros comenzaron a participar y las asociaciones de India y Nueva Zelanda fueron admitidas. Hubo también homenaje a Florencia Nightingale que había fallecido en agosto de 1910, y por ello, Ethel Fenwick propuso que se instituyera una fundación con su nombre para el desarrollo de la educación de enfermería, a fin de que los enfermeros de todo el mundo pudieran prepararse debidamente y seguir su ejemplo. En la ocasión fue recordado, también, que el local del evento, Colonia, estaba muy próximo de Kaiserwerth, lugar donde Florencia había aprendido los fundamentos iniciales de la enfermería. En Colonia fue elegida la primera americana, Annie Goodrich, como presidenta del CIE.

La reunión siguiente sería en San Francisco, en 1915, pero en setiembre de 1914, varias organizaciones informaron que no podrían enviar representantes debido al inicio de la 1a. Guerra Mundial (1914-1918). De hecho, sólo pudieron comparecer los representantes de Inglaterra, Australia y Holanda, viajando todas por navío. Lavinia Dock, que continuaba como secretaria honoraria del CIE, no pudo comparecer porque estaba totalmente involucrada en la lucha por el derecho del voto femenino en su país, lo que se obtuvo en 1919.

Como se ve, las raíces del CIE están muy ligadas al sufragio femenino, a los derechos de la mujer y reformas sociales para fortalecer la profesión y tener voz en los círculos políticos y de poder. De cualquier forma, además de la convención americana de los enfermeros hubo la reunión para tratar los asuntos del CIE, cuando fue elegida la danesa, Henny Tcherning, como presidenta del CIE, para el mandato siguiente que sería de 1915 a 1919, pero solo terminó en 1922, porque debido a la Primera Guerra Mundial, no hubo posibilidad de que el CIE convocara una reunión. En este último año de su mandato fue realizada, en Copenhague, la reunión del Gran Consejo, como era llamado anteriormente el CRN, siendo elegida como presidenta del CIE Sophie Mannerheim, de Finlandia.

En 1925 en Helsinki, capital de Finlandia, fue realizado el congreso siguiente del CIE, cuando pasó su cargo a Nina Gage, una americana nacida en Nueva York, donde también hizo el curso de enfermería. Como ella estudió el chino por muchos años, se identificaba con ese pueblo, y trabajaba desde 1909, como Directora de una Escuela de Enfermería en Hunan, China, donde pasó casi toda su vida. Cuando fue elegida quería renunciar, dada la distancia y las dificultades para estar en Europa, donde la mayoría de las acciones tenían que ser tomadas, pero las enfermeras chinas la instaron a no hacerlo, pues sentían orgullo de tener a la presidenta del CIE viviendo en China, donde pretendían realizar el congreso de 1929, lo que no ocurrió debido a problemas políticos y acabó siendo realizado en Montreal.

Luego del Congreso de 1925, aún en Finlandia, la Junta Directiva se reunió con Nina Gage para discutir las cuestiones internas administrativas de la organización, época en que las líderes del CIE percibieron que esta organización, ya con 24 miembros asociados, estaba 
creciendo mucho y necesitaba tener su propia sede y personal remunerado.

Así, en octubre de 1925, Christiane Reimann, enfermera danesa, asumió el cargo de Secretaria Ejecutiva y transfirió la sede del CIE de Londres a Ginebra. Durante los tres primeros años, ella continuó haciendo trabajo voluntario, hasta que las finanzas permitieron que fuera remunerada. La elección de Suiza como sede de la organización fue por causa de la condición de neutralidad de ese país frente a los conflictos bélicos y el alto valor de cambio de la moneda suiza. Las guerras en las que Inglaterra se había metido hasta entonces, constituían motivos suficientes para no recomendar a Londres como el mejor local para ser sede de una organización internacional. Además que otras organizaciones, como la OIT fundada en 1917, tenían ahí su sede, así como la Cruz Roja Internacional y la Liga de las $\mathrm{Na-}$ ciones (antes de ser transformada en ONU) y Christiane, desde el comienzo, trató de establecer buenas relaciones con esas entidades. En 1926 creó una revista, actualmente llamada International Nursing Review. En noviembre de 1927, Christiane organizó una conferencia en Ginebra, y aún sin haberse realizado la clausura, ella ya estaba organizando el Congreso de Montreal para julio de 1929. En ese evento fue elegida la francesa Leonie Chaptal, como presidenta y a partir de ese año quedó establecido que los congresos del CIE serían cuadrienales y que en los intervalos ocurrieran reuniones del CRN.

La localización de la sede en Ginebra dificultaba los trabajos del CIE pues la mayoría de las actividades tenían que ser conducidas a partir de Londres y el staff se sentía profesionalmente aislado, pues pocas enfermeras visitaban el CIE en una ciudad pequeña y sin atractivos, como era Ginebra. Se tomó la de- cisión del retorno de la sede para Londres, lo que ocurrió en junio de 1937. La inauguración de la nueva sede coincidió con la realización del tercer congreso cuadrienal, habiendo sido elegida presidenta del CIE la americana Effie Taylor y contó con la presencia de Ethel Fenwick, ya con 80 años de edad.

El tema más importante tratado en esa reunión fue, probablemente, el informe de la Comisión de Educación que recomendaba que el CIE desarrollara un amplio estudio sobre la educación de enfermería. Tal estudio no pudo ser concretizado debido a la inestabilidad política en Europa que resultó en el inicio de la $2^{\mathrm{a}}$. Guerra Mundial en el mismo año. Londres en la mira de los bombardeos, obligó a las líderes del CIE a transferir rápidamente los documentos y archivos importantes de la sede hacia la casa de amigos en Cambridge, en el interior de Inglaterra. No sintiendo, aún, seguridad fue decidido llevar tales documentos a los Estados Unidos, que eran neutros, ya que todavía no habían entrado en la guerra. Tomada la decisión, la americana Calista Banworth, que había sido nombrada Secretaria asistente del CIE, se embarcó en navío el día 12 de octubre de 1939, en viaje de un mes, llevando toda la documentación esencial e histórica de la organización a la ciudad de New Haven, Connecticut, en los Estados Unidos.

La Asociación Americana de Enfermeras ayudaba en la manutención de esta sede, que fue transferida a Nueva York, en 1945. La participación de los Estados Unidos en la guerra mundial interrumpió las actividades del CIE, que en su total duró diez años.

Terminadas las hostilidades y con el fin de la guerra, las líderes de enfermería se encontraron en septiembre de 1946, en Londres, en la sede del Royal College of Nursing. En esta reunión se tomaron algunas decisiones im- 
portantes sobre la retomada de actividades, la realización de congresos cuadrienales y el retorno de la sede a Londres, lo que ocurrió en noviembre de 1947, con la americana Virginia Arnold quien acompañó personalmente esta mudanza, en navío. Ella permaneció aún por algún tiempo, como secretaria en ejercicio, hasta que la inglesa Daisy Bridges fue nombrada, en abril de 1948, en cuyo cargo permaneció hasta fines de 1961. En 1947 ocurrió el congreso en Atlantic City (EUA), siendo elegida como presidenta Gerda Höjer, de Suecia, quien tuvo que quedarse seis años en el cargo, ya que la elección siguiente ocurriría en 1953. En 1949, en la ciudad de Estocolmo, fue realizada una conferencia especial para celebrar el cincuentenario del CIE. En esa conferencia fue decidida la realización del X Congreso Cuadrienal, en 1953, en Brasil, por primera vez, en un país de América del Sur, pues anteriormente todos esos eventos ocurrían sólo en los países del hemisferio norte. En 1973, el XV Congreso del CIE fue realizado en la ciudad de México, el segundo evento de este porte en país latinoamericano, y en 1993, en Madrid, España.

\section{Las sedes del CIE y su retorno a Ginebra}

A partir de 1948, finalmente el CIE consiguió mantener un trabajo estable y continuo con Daisy Bridges al frente. En octubre de 1955, la Junta Directiva del CIE decidió adquirir sede propia, una casa de cuatro pavimentos en Westminster, para donde el CIE fue transferido.

Nueva y definitiva mudanza de sede ocurrió en 1966, de esta vez retornando a Ginebra, no solamente porque la sede quedó pequeña para las actividades que precisaban ser realizadas, sino también porque una sede en Ginebra sería menos onerosa que en el centro de Londres. Además del factor costo, había otra

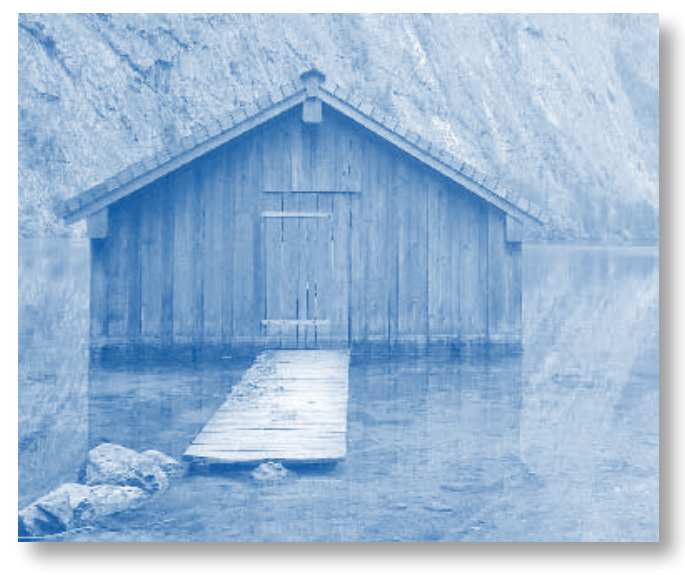

cuestión esencial para el CIE, como federación internacional. Para seguir como portavoz de los enfermeros de todo el mundo, tendría que trabajar próximo a otras organizaciones internacionales, como la OMS, creada en 1948, así como la OIT y la Cruz Roja Internacional, todas asentadas en Ginebra. El CRN durante el Congreso de 1965, en Frankfurt, aprobó esa transferencia.

Daisy Bridges partió del CIE a fines del año de 1961 y desde 1962, ocuparon el cargo de directora ejecutiva varias enfermeras, todas permaneciendo entre dos a tres años, hasta que la licenciada Constance Holleran, con larga experiencia de trabajo en la Asociación Americana de Enfermeras fue indicada y aceptada por la Junta Directiva. Holleran asumió el cargo en abril de 1981, donde permaneció por 15 años hasta fines de 1995, cuando se jubiló. Este fue un período de gran estabilidad y productividad de la organización, pues es la directora ejecutiva con su staff la que implementa las decisiones de la Junta Directiva, que como ya se ha mencionado se reúne una vez al año y el CRN sólo una vez cada dos años. En 1996, asumió el cargo la canadiense Judith Oulton, también con larga experiencia en la Asociación Canadiense de Enfermeros. Finalmente, en octubre de 2008, asumió el Sr David Benton, de Escocia, por primera vez un enfer- 
mero varón al frente del Consejo Internacional de Enfermeras.

\section{Cambios estructurales}

Como referido anteriormente sobre los idiomas del CIE, a partir de las elecciones de 1993, cuando asumió la presidencia la americana Margretta Styles, la Junta Directiva pasó a contar con la traducción simultánea entre inglés, francés y español. Fue también en este mandato que surgieron diversos problemas políticos en distintos países que se desmembraban y hasta de Brasil, que no se desmembró, pero ocurrió la transferencia de afiliación con el CIE, en 1997, de la ABEn, que era miembro desde 1929 al COFEN. De hecho, entidades con afiliación compulsoria de profesionales, tales como los colegios y consejos, en general, cuentan siempre con la ventaja de tener más miembros que una institución de afiliación voluntaria o facultativa.

Sin duda al CIE le interesaba, y siempre le interesó, también el ingreso del mayor recurso financiero, pues la cotización está basada en el cálculo per cápita sobre el total de enfermeros afiliados. Para cada enfermero asociado o inscrito, la organización debe pagar un valor determinado de acuerdo con una tabla anual del Banco Mundial sobre el Producto Bruto Interno (PBI) del respectivo país.

El presupuesto del CIE se compone del resultado de esas cotizaciones o contribuciones hechas por las organizaciones afiliadas (60 a $70 \%$ ), fondos para proyectos (30 a $35 \%$ ) y venta de publicaciones (5\%). Entre las ANEs con más de cien mil miembros que han contribuido con sus cotizaciones están las de Japón, Reino Unido, España, Estados Unidos, Australia, Canadá y Brasil.

Otro tema político de larga duración fue el relacionado con el apartheid en África del Sur.
A pesar de que la Asociación Sud Africana de Enfermeras se afilió en 1922, por causa de esta discriminación racial tuvo que pedir exclusión del CIE, en CRN de México, en 1973. Solamente con el fin del apartheid y la elección de Nelson Mandela, como presidente de la República, la afiliación de la ANE sudafricana con el CIE fue restablecida, en 1995, y oficialmente introducida en 1997. La antigua organización fue sustituida por la DENOSA (Democratic Nursing Organization of South Africa), cuya primera presidenta, Philda Nzimande, fue elegida para integrar la junta directiva del CIE, de 2001 a 2005 y re-elegida para 2005-2009.

Una nueva tradición fue introducida a partir de 1985. Christiane Reimann, la primera secretaria ejecutiva remunerada del CIE, de 1922 a 1933, falleció habiendo dejado sus bienes en testamento para que fuera instituido un premio internacional a ser otorgado por ocasión de los congresos cuadrienales a enfermeras (os) que, en los años inmediatamente anteriores a la fecha del premio, hayan hecho considerable esfuerzo dentro de la profesión o para la profesión de enfermería, a través de la investigación académica o de la práctica de enfermería en beneficio de la humanidad. Christiane Reimann, nacida el día 6 de mayo de 1888, en Dinamarca, tuvo una educación refinada. Era licenciada en ciencias y tenía el título de magister en enfermería por la Universidad de Columbia, Nueva York, lo que era raro en la época. En esta Universidad mantuvo estrecho contacto con docentes americanas notables, como Adelaide Nutting e Isabel Stewart, que mucho influyeron en su carrera. En 1922 fue elegida como secretaria ejecutiva, por recomendación de Lavinia Dock a quien sustituyó. En 1925, ella fue reelegida para el mismo cargo que pasó a ser remunerado y permaneció en él hasta diciembre de 1933. Christiane falleció el 
12 de abril de 1979, con casi 92 años de edad. Después de su muerte, sus abogados juntamente con el CIE establecieron los criterios y condiciones para la concesión del premio, de acuerdo con su última voluntad. Los intereses del fondo instituido serian administrados por la Organización Danesa de Enfermería, que serían retirados cada cuatro años para ser entregado a la persona propuesta por la ANE y evaluada por el CIE entre los candidatos así indicados. A este premio se dio el nombre de Premio Christiane Reimann, un homenaje a la instituidora, sin embargo también es conocido como el Premio Nobel de Enfermería. La primera enfermera a merecer este Premio fue la enfermera americana Virginia Henderson, en 1985, por su enorme contribución con la teoría de los cuidados básicos de enfermería, traducida a más de 20 idiomas. El monto del premio es alrededor de $\$ 10.000,00$ dólares americanos, dependiendo del valor de cambio entre la moneda danesa y el dólar. El último a recibir este premio fue Máximo Gonzales Jurado, de España, en 2009.

\section{Importancia del CIE en la historia de la en- fermería}

La creación del CIE fue extremamente importante para toda la enfermería mundial. Fue un marco donde se empezó el desarrollo de mujeres que se organizaban, no en nivel de sus ciudades, pero de sus países buscándose el apoyo de compañeras de profesión, y así fortaleciendo la enfermería. Tal iniciativa pionera es sorprendente en una época que no se disponía de los medios de comunicación, ni de transporte hoy existentes, además de las dos guerras mundiales que enfrentaron, cuando para proteger los documentos y archivos, las enfermeras viajaron de un sitio al otro a veces por meses como ocurrió con el viaje de Ingla- terra para los Estados Unidos. Tal hecho solo ya tiene un valor extraordinario, pues ellas estaban defendiendo y protegiendo un patrimonio cultural e histórico de la memoria de enfermería. Fueron, pues mujeres visionarias que arriesgaban su seguridad y conforto en beneficio del ideal de tener y mantener la fuerza de una organización internacional.

Pero, antes de comenzar a mejorar la situación profesional de enfermería, ellas sintieron que era necesario que las mujeres tuviesen derechos de ciudadanía que garantizase su participación en las decisiones políticas de sus respectivos países. Las pioneras del CIE incentivaron para que se luchase por el derecho al voto femenino. Es importante recordar que cuando fue creada, en 1899, solamente en Nueva Zelanda las mujeres tenían derecho al voto, desde 1893, y una de las líderes de la marcha por este derecho había sido la enfermera Grace Neil. En Estados Unidos Lavinia Dock, otra líder pionera del CIE también luchó por este derecho, reconocido finalmente en 1919.

Como antes referido fue a partir del Consejo Internacional de Mujeres (CIM) que se creó el CIE, en una época en que el papel de la mujer aun se reducía al hogar, una tradición doméstica mantenida por muchos siglos. Las mujeres estaban excluidas no solamente de las tareas de gobierno, de política y hasta del comercio y de la mayoría de actividades profesionales.

La Iglesia Católica a través de monasterios y de la vida religiosa permitió a las mujeres estudiar y se dedicar al cuidado de enfermos y pobres, como fueron las Hijas de la Caridad de San Vicente de Paul (1576-1660), alrededor de 1600, cuando la miseria y la enfermedad por las constantes guerras estaban aniquilando Francia. Pero, era practicada como obra de misericordia o caridad y no como profesión. 
Además de los religiosos que cuidaban de enfermos había también personas sin ningún preparo técnico o estudio específico, ni de comportamiento social o moral, que cuidaban empíricamente de los enfermos. Esta fase ocurrió principalmente en Inglaterra y el escritor Charles Dickens inmortalizó la figura de Sarey Gamp, como la anti-enfermera o la pre-profesional de enfermería. Todo esto crió la mala fama por siglos contra la función o profesión enfermera.

Florencia Nightingale surgió para cambiar esta situación social y transformar la obra de caridad de cuidar de los enfermos en actividad profesional de enfermería, digna y respetada. Era un largo proceso, que sigue todavía en algunos países, pues es difícil cambiar actitudes de siglos en décadas. Pero se puede decir que se avanzó muchísimo principalmente a partir de las investigaciones, estudios científicos y publicaciones de enfermeros. La difusión del modelo de la enfermería moderna fue implantada a partir de Inglaterra y Estados Unidos para todo el mundo.

De hecho, la incidencia del CIE fue instrumental e importante en aquel momento histórico de 1899, cuando la enfermería estaba todavía dando sus primeros pasos para la efectiva profesionalización en la mayoría de los países occidentales. Aún que la actividad era considerada importante para la supervivencia de niños y enfermos, nunca tuvo gran relevancia pues las ciencias biológicas, médicas y de salud eran muy incipientes y no había tampoco recursos tecnológicos. Pero, la creación del CIE fue y sigue siendo instrumental para la difusión de la enfermería moderna, universitaria y competente, y para estimular la unión de los profesionales alrededor de una organización fuerte y representativa que pueda influir en la política social, de salud y de enfermería, así como en las normas profesionales y socio-económicas en sus respectivos países.

Como porta-voz de los enfermeros en el mundo el CIE ha desarrollado su trabajo con verdadera diplomacia en el trato de todos los temas profesionales, procurando hacer avanzar la profesión e influenciar las políticas de salud, como está definida en su misión. En todos los países, hubo siempre algunas enfermeras pioneras que asumieron la lucha para mejorar las condiciones de salud de su pueblo a través de la atención de enfermería. Cada país tiene que rescatar e identificar los nombres de estas pioneras y ponerlas en el lugar que merecen dentro de la profesión, documentándose los hechos y conquistas para que las generaciones futuras puedan conocer a sus líderes nacionales y sirvan de inspiración para conquistas mayores para la profesión y para el reconocimiento profesional de los enfermeros.

La difusión y socialización del CIE será beneficiosa también a los profesionales de Historia de la Enfermería, pues por primera vez, el tema historia de la enfermería apareció como una línea especifica de trabajos y estudios en su conferencia internacional de 2011. Se espera que los enfermeros se sientan estimulados a presentar resultados de sus investigaciones y estudios históricos también en este foro que reúne enfermeros de más de cien países. Así, el campo de Historia de la Enfermería podrá crecer y alcanzar límites geográficos mucho más amplios y profundos. Si estamos convencidos de que la Historia de Enfermería es fundamental para que se conozca su propia profesión y su identidad profesional, la socialización de estos conocimientos será también fundamental para la valoración de los estudios e investigaciones por parte de nuestros propios colegas de profesión. 


\section{CONSIDERACIONES FINALES}

Esta trayectoria histórica del CIE es un recordatorio para los enfermeros en general y también para los que se dedican a un aspecto específico de la historia de la profesión. Podemos y debemos tornar este campo de nuestros estudios más amplio y universal, quizás conquistar más adeptos e investigadores para la historia si nos aliamos con el universo mayor de enfermería. El apoyo de nuestros propios colegas de otras especialidades también es importante para tener la historia de enfermería más valorada en las escuelas de enfermería tenerla incluida y así mantenida en los currículos y planos de estudios. Colegas de profesión precisan estar convencidos de la importancia de la historia para la formación de nuestra identidad profesional, hecho fundamental para ampliarse el universo de enfermería. Quien somos, dónde venimos, para dónde vamos, qué queremos, qué buscamos son interrogantes que necesitan de respuesta que las dará la historia de enfermería. Quien de nosotros no está sintiendo que las modernas especialidades basadas en las nuevas tecnologías están cada vez más agresivas, ocupando más espacios y horas o días de los currículos? De forma clara o sutil cada uno de nosotros tiene que luchar para mantener su carga horaria de clases en los cursos de enfermería para hacer sobrevivir la Historia de Enfermería dentro de miles de horas del curso.

También nosotros, estudiosos de la Historia de Enfermería, tenemos que salir de nuestro mundo histórico y chapuzar en un universo mayor de enfermería para aumentar nuestros espacios y buscar más interesados y más apoyo por el tema.

Para tanto, deberíamos conocer más, participar más del CIE en sus eventos bienales, escribir artículos y textos sobre el tema para la revista del CIE, de amplia circulación internacional, para presentar trabajos en sus conferencias y congresos. Así podremos también ampliar la comunicación con otros especialistas de enfermería y cruzar informaciones con todos. De hecho debería ser motivo de orgullo para todos los enfermeros tener el CIE y poder afirmar que su organización internacional de enfermería es más antigua que la propia OMS (que existe poco más de 60 años), la ONU y otras organizaciones de carácter mundial, hasta aún la Organización Internacional del Trabajo (OIT), creada en 1917, antes de la ONU.

En un mundo globalizado no podemos restringir nuestro campo de acción a unos pocos y pequeños grupos de enfermeros. Así nos condenaríamos a ser siempre pequeños. Tenemos que fortalecer las identidades culturales de pueblos que pertenecen a una raíz histórica muy similar y criar una comunidad internacional que puede hincar la bandera ibero-americana en el espacio mundial de la historia de la enfermería. Un paso fue dado en 2009 cuando tuvimos coraje de crear la Federación Ibero-americana de Historia de la Enfermería, en Lisboa, Portugal, el 9 de octubre. Carecemos todavía del registro notarial a ser providenciado, en España, a partir de la aprobación de sus Estatutos. Para ser una federación, la nueva organización debe tener otras entidades en el mundo ibero-americano. Enfermeros y otros profesionales de Brasil, dentro del principio de interdisciplinaridad, trataron de crear legalmente una entidad llamada Academia Brasileña de Historia de la Enfermería (ABRADHENF), el día 13 de agosto de 2010 (centenario de la muerte de Florencia Nightingale) cuya junta directiva cuenta con miembros procedentes de cuatro estados (o provincias) del país. En Portugal fueron creadas la Sociedad Portuguesa de Historia de la Enfermería y la 
Asociación Nacional de Historia de la Enfermería (ANHE), en Lisboa, Portugal. En julio de 2011 fue creada la Asociación Histórico Filosófica del Cuidado y la Enfermería en México (AHFICEN). En Colombia ya existía la Red Ibero-americana de Historia de la Enfermería.

Todas estas entidades estarán representadas en el evento de Alicante, que tiene por lo tanto una importancia histórica mayor, pues debe aprobar los Estatutos de la Federación Ibero-americana de Historia de la Enfermería, y así fortalecer esta área del saber de la profesión enfermera.

\section{BIBLIOGRAFÍA}

- Bridges, DC (1967) A history of the International Council of Nurses, 1899-1964, the first sixty-five years. JB Lippincott Co, Philadelphia.

- Brush BL, Linaugh JE, Boschma G, Rafferty AM, Stuart M, Tomes NJ (1999) Nurses of all nations - a history of the International Council of Nurses, 1899-1999. Lippincott, Philadelphia.

- Felizzia ASM (2008) El surgimiento de la enfermería como profesión de mujeres. El ser enfermero, Buenos Aires, Argentina. 1(2):31-7.

- ICN (2009) Constitution, amended, Geneva, Switzerland.
- ICN (2009) Dues payment report. In: Council of National Representatives, Durban, South Africa, 27-30: 200201.

- McGann S, Crowter A, Dougall R(2009) A history of the Royal College of Nursing: 1916-1990. A voice for nurses. Manchester University, UK.

- Malchau S (2001) Christiane Reimann, 1888-1979, the lady behind the "Nobel Prize" in nursing. The Danish Nurses' Organization, Denmark.

- Quinn S (1989) ICN - past and present. Scutari Press, England.

\section{NOTAS.}

1 Grace Neil fue una de las líderes del movimiento popular en Nueva Zelanda por el derecho de voto de la mujer. De hecho, este país fue el primer del mundo a dar a la mujer el derecho de voto, en 1893. In Stodart K Suffrage: a pioneer for nursing. Nursing New Zealand, Wellington, 1993. 1(6): 28-9.

2 ICN Constitution, amended 2009, International Council of Nurses, Geneva, Switzerland.

3 Se recuerda que el CIE ya tuvo como presidentas una enfermera francesa, Leonie Chaptal, una de Bélgica Marie Bihet, y una de Canadá, Alice Girard. Evidentemente, Francia tiene como idioma oficial el francés, sin embargo, los otros dos países tienen una parcela de la población que tiene el francés como idioma oficial.

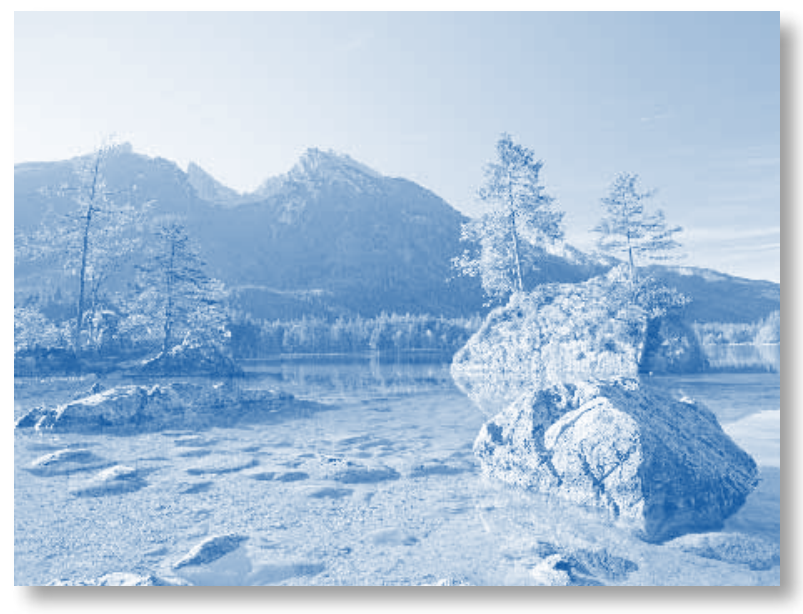

